\title{
A comprehensive review on COVID-19: What we know and how to treat against the novel coronavirus
}

\author{
Jiacheng Wang ${ }^{1, \dagger}{ }^{*}$, Xianhao $\mathrm{Xu}^{2, \dagger}$, Zihan $\mathrm{Xu}^{3, \dagger}$ \\ ${ }^{1}$ College of Life Science, Nankai University, 300071 Tianjin, China \\ ${ }^{2}$ Wuhan Britain-China School, 430000 Wuhan, China \\ ${ }^{3}$ International Department, Shandong Experimental High School, 250001 Jinan, Shandong Province, China \\ †These authors contributed equally.
}

\begin{abstract}
COVID-19 emerged in Wuhan, China, at the end of 2019 and then soon evolved into a global pandemic. The novel coronavirus inducing this pandemic is under extensive study held by researchers all over the world. We give out a comprehensive review of what we have known about this novel coronavirus, including the pathogenesis. Passive immunity, different strategies, and targets for vaccine development and antiviral drugs are introduced as therapeutic strategies. At last, many other properties of SARS-Cov-2 are discussed.
\end{abstract}

\section{Introduction}

Starting from the end of 2019, when the new coronavirus was first discovered, and by May 20th, 2021, the virus has swept across all continents with a cumulative total of 166 million cases and 3.44 million deaths[1]. The concentration of cases moved from China in the beginning to the United States, Europe, and other regions to India today. In the process of this virus spreading, the new virus even began to mutate accordingly, forming several variants such as B.1.1.7, B.1.351, B.1.427 lineages. Such mutations allowed the virus to gain more opportunities to spread and replicate, aggravating the number of infections in the world and making it more difficult for the world to break away from the viral quagmire[2].

However, mankind is also trying to use its experience and technology accumulated over decades to ease the current tension in this game. Since last year, Russia, China, the United States, the United Kingdom, and other countries have launched their vaccines. By now, more than 1.62 billion doses have been given to the public worldwide[3].

\section{COVID Pathogenesis}

\subsection{Virus entry}

'SARS-CoV-2 is transmitted mainly through air droplet, human-to-human contact, and potential in faecal-oral. Primary viral replication is presumed to occur in the mucosal epithelium of the upper respiratory tract (nasal cavity and pharynx), with further multiplication in the lower respiratory tract and gastrointestinal mucosa, giving rise to a mild viremia. Few infections are controlled at this point and remain asymptomatic. Some patients have also exhibited non-respiratory symptoms such as acute liver and heart injury, kidney failure, diarrhoea, implying multiple organ involvement. ACE2 is broadly expressed in the nasal mucosa, bronchus, lung, heart, oesophagus, kidney, stomach, bladder, and ileum, and these human organs are all vulnerable to SARSCoV-2. Recently, potential pathogenicity of the SARSCoV-2 to testicular tissues has also been proposed by clinicians, implying fertility concerns in young patients.'[4]

Before SARS-CoV-2 enters the target cell, the virus needs to recognize a specific surface protein molecular target on the cell, and the specific protein is ACE-2 which stands for 'Angiotensin Converting Enzyme-2'. For a typical type II pneumocyte cell, the SARS-CoV-2 virus will bind to ACE-2 protein. With the facilitation of TMPRSS protein, the virus can attach to the cell and fuse with it, leading to endocytosis, letting the virus enter the cell's cytosol by vesicle transportation. Once into the vesicle, the virus encodes and releases a particular nucleic acid out of the actual viral envelope. The nucleic acid strand is a positive-sense singlestranded RNA, and once it's been released to the cytoplasm, it will either bind to free or rough endoplasmic reticulum ribosomes. Then, RNA gets translated and makes particular polyproteins used to make spike proteins, hemagglutinin esterase proteins, $\mathrm{M}$ proteins, envelope proteins, etc. Besides, RNAdependent RNA polymerase is produced simultaneously,

\footnotetext{
* Corresponding author: jiachengwang@mail.nankai.edu.cn
} 
taking RNA to produce even more viral positive-sense single-stranded RNA molecules. The newly-formed viruses are packed with lipid bilayers produced by the Golgi body, and they will fuse with the cell again via exocytosis, resulting in cell death.

\subsection{Infection}

When type II pneumocyte cells are damaged, different types of molecules, including interferons $\alpha$ and $\beta$ are released. Interferons take charge of transmitting the signal of virus presence to the surrounding uninfected cells. In a further step, antiviral peptides are secreted as a part of the immune reaction.

What's more, whenever the cells are damaged, they will start releasing damage-associated molecular patterns (DAMP) and inflammatory cytokines. Cytokines alert an alveolar macrophages vicinity, and macrophages continue to secrete inflammatory mediators including interleukin-I, interleukin-6, interleukin-8, TNF- $\alpha$, interferon $\gamma$. Those molecules then move into the pulmonary capillary area, causing the wall of the capillary more permeable, leading to fluid leakage into the alveoli, forming edema, making patients short breaths.

\section{Therapeutic strategies}

Due to the COVID-19 outbreak in Wuhan, China, and the subsequent rapid spread worldwide, vaccination has been drawing tremendous attempts as a promising method to diminish this novel coronavirus. What's more, there are currently no specific antiviral drugs against SARS-Cov-2, which adds to the importance of vaccine development and administration. Strategies for vaccine development usually include DNA-based vaccine, RNAbased vaccine, live-attenuated vaccine, inactivated virus vaccine, recombinant viral vaccine, and subunits vaccine. Apart from vaccination, other antiviral methods like passive immunization also worth attention.

\subsection{Passive immune therapy}

In the early phase of the COVID-19 pandemic, there was no efficient treatment against this novel coronavirus, so the clinical treatments mainly encompass supportive care and therapies mitigating the symptoms[5]. Thus, passive immune therapy played a significant role during the early phase of the COVID-19 pandemic.

Convalescent plasma from recovered patients contains specific antibodies against a pathogenic antigen. Therefore, when convalescent plasma is injected into the infected patients, the antibodies work to neutralize antigens. Convalescent plasma transfusion that entails passive immunity emerged in the late 19th century and has been adopted in treatments against various pathogens, including SARS-Cov, MERS-Cov, Ebola, and COVID19. Back in the 1890 s, whole blood or plasma was used in treatment against microbe infections [6]. Later, Emil Adolf von Behring (1854-1917) won the first Nobel Prize in 1901 to discover serum therapy as a viable method to treat diphtheria [7, 8]. Besides, Emil Adolf von Behring and a Japanese physician Shibasaburo Kitasato (1852-1931), prepared serum from immunized rabbits to treat tetanus[9, 10]. There is also a longstanding history of convalescent plasma used as a treatment against virus-caused infectious diseases. For example, it is documented that convalescent plasma therapy (CPT) was employed in the 1918 Spanish flu pandemic[11]. An analysis demonstrated reduced mortality during the Spanish flu pandemic after the use of convalescent plasma products[12]. In more recent cases like MERS-CoV, H1N1 and H5N1 avian flu, Ebola, and SARS-Cov, CPT showed satisfying outcomes in treating patients[6].

CPT provided the most promising treatment that entailed rapid and effective passive immunity while antiviral drugs and vaccines were under clinical trials. Various cases and studies proved the effectiveness of CPT. Fischer et al. reported that after convalescent plasm transfusion, viral load decreased within 12 days in about 5 patients[13]. Additionally, Duan et al. reported in 2020 that 9 severely ill COVID-19 patients received CPT and showed reduced viral load and inflammation[14].

CPT's advantages in the early phase of the pandemic are prominent, such as immediate and effective immunity and cost-effectiveness. It bypasses the use of a ventilator which is extremely limited at the peak of the COVID-19 pandemic. However, CPT also has some drawbacks in clinical practice. For example, CPT only maintains a short-term antibody level in human bodies[15]. Additionally, if few, some cases are reporting adverse effects after convalescent plasma transfusion, such as anaphylactic reaction[6].

\subsection{Vaccination strategies}

\subsubsection{Live-attenuated vaccine}

Live-attenuated vaccines have been proved effective by the cases of various bacterial and viral pathogens, like Mycobacterium Tuberculosis and human papillomavirus[16, 17]. The biggest problem of the liveattenuated vaccine lies in the possibility of regaining the virulence, which used to happen in the oral vaccine of poliovirus[18]. Despite the risk, it is still tempting to use attenuated pathogen as a vaccine since it displays all possible viral antigens to the immune system. Notably, researchers are working to mitigate the risk of attenuated pathogens regain their virulence through genome editing. For example, deletion of the E protein-encoding gene abrogates the virulence of coronavirus, as was reported by M.L. DeDiego et al. [19].

\subsubsection{Inactivated virus vaccine}

The inactivated vaccine is also known as the whole killed virus (WKV), which contains viral particles whose abilities to infect and replicate are removed by radiation or chemicals. WKV can also present the same epitope as natural viral particles while lacking the ability to cause disease. It is thought that the simple preparation of WKV 
makes it one of the most popular vaccines in the market. In China, three vaccines in the market are inactivated virus vaccines.

\subsubsection{DNA/RNA-based vaccine}

The nucleic acid vaccine only contains DNA or mRNA encoding viral antigens. After being taken up and expressed by cells, these antigens are processed by protease inside cells and presented through the MHC class I pathway (Figure 1). Compared with the liveattenuated vaccine, one of the prominent advantages of the nucleic acid vaccine is safety since attenuated pathogens may revert to their original form and cause disease. The first DNA vaccine is the HIV-I vaccine, which has displayed the safety and protective efficacy of the DNA vaccine[20]. Also, to achieve herd immunity, many vaccines should be produced and administered, which can be fulfilled by the ease in mass manufacturing of nucleic acid vaccine for it circumvents the complicated process of protein purification.

\subsubsection{Recombinant viral vaccine}

Recombinant viral vaccines utilize carrier viruses like adeno or pox virus as carriers of modified genetic materials like foreign DNA encoding viral antigens of another virus. This category of vaccine can be classified as replicating and non-replicating viral vectors. Since recombinant virus stays active inside human bodies like their natural counterparts, recombinant viral vaccine usually elicits a strong and long-lasting immune response, which means people only need one injection to get immunized. Recently, an adenovirus-based recombinant viral vaccine developed by Wei Chen from the Academy of Military Sciences PLA China cooperating with CanSinoBIO has been permitted to come into the market in China.

\subsubsection{Subunits vaccine}

Protein subunits vaccines only contain parts of the virus that can elicit an immune response. Protein subunits vaccine can be further divided into protein-based subunits vaccine, polysaccharide subunits vaccine, and conjugated subunits vaccine. Considering the constituents of subunits vaccine only include synthetic polypeptides and recombinant proteins that serve as antigens, there is no need to worry about the side effect of vaccines like the capability of promoting disease.

\subsubsection{Targets for vaccine development}

Spike protein (S protein) has been considered the most promising target for vaccine development against COVID-19. Like other coronavirus $S$ protein, the $S$ protein of COVID-19 can be cleaved by protease into S1 and S2 subunits. The process of COVID-19 entering the cells is mediated by $\mathrm{S}$ protein, which includes several steps: attaching to the cell surface, conformational alteration of $\mathrm{S}$ protein, proteolysis of the $\mathrm{S}$ protein, and releasing the S2 subunits, which then causes membrane fusion and cellular entry of COVID-19[21]. As an essential molecule for viral entry, $\mathrm{S}$ protein has been the subject of vaccine developments. On January 10th, 2020, the full genetic sequence of $\mathrm{S}$ protein has been released, facilitating the development of a vaccine targeting $S$ protein[22]. Various vaccines are designed to target $S$ protein, including DNA vaccines, viral vector-based vaccines, and subunit vaccines[23].

Apart from the $\mathrm{S}$ protein, some other viral proteins can also be potential targets, like Nucleocapsid (N) protein and Membrane (M) protein.

The SARS-Cov-2 N protein is more conserved than $\mathrm{S}$ protein and can induce immune responses in hosts[24]. $\mathrm{N}$ protein functions in viral particle assembly and the regulation of viral gene transcription. According to Carlson et al., the unmodified oligomeric state of the $\mathrm{N}$ protein helps with viral assembly while the phosphorylated $\mathrm{N}$ protein presenting liquid-like properties helps with gene transcription[25].

$\mathrm{M}$ protein is vital for the virus budding process. In a recent study, multiple CD4-restricted T-cell epitopes are identified within a highly conserved region of $M$ protein[26], which implies that $M$ protein could be a promising target for vaccine development. 


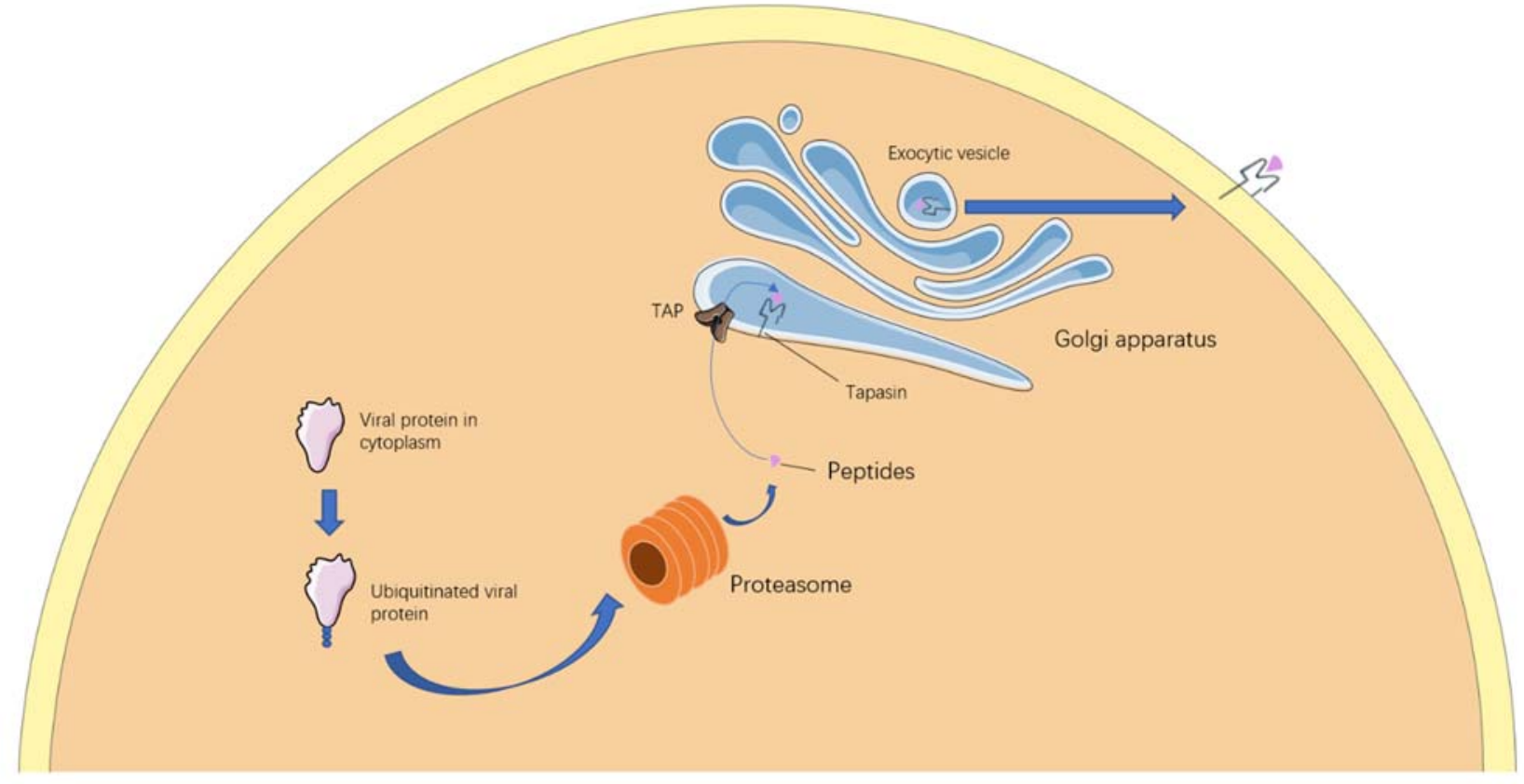

Figure 1. Schematic illustration of MHC Class I pathway

\subsection{Antiviral drugs}

Due to the long period of developing a new drug for an emerging virus, drugs used to treat COVID-19 are mainly existing drugs used to treat previous virusinduced diseases. For instance, Ribavirin is a synthetic guanosine analog prodrug that interrupts viral RNA synthesis by interfering RNA dependent RNA polymerase. Ribavirin was firstly commercialized in the early 1980s and was originally proposed to treat the respiratory syncytial virus in children. As a broadspectrum antiviral drug, ribavirin effectively treats Crimean-Congo hemorrhagic fever, Hantavirus infection, Lassa fever, and Venezuelan hemorrhagic fever[27]. Aside from ribavirin, favipiravir and remdesivir also interfere with RNA replication by impairing the function of viral RdRp. Hydroxychloroquine and chloroquine halt viral entry by impeding the glycosylation of ACE2[28], which is the receptor mediating the entry of SARS-Cov2. Also, Hydroxychloroquine and chloroquine can increase the $\mathrm{pH}$ value inside the endosome, so they can be used to treat other viruses that depend on the acidic environment in the endosome to enter the cytosol.

\section{Structure}

SARS-CoV-2 is a large, enveloped, positive-stranded RNA virus and appears like a crown under the electron microscope. Compared with other known coronaviruses, SARS-CoV-2 shares sequence similarity with them, including MERS-CoV (50\%), SARS-CoV (79\%), batSL-CoVZC45, and bat-SL-CoVZXC21 (80\%)[29]. Its genome contains several open reading frames encoding for four structural proteins: spike (S), envelope (E), membrane $(\mathrm{M})$, and nucleocapsid $(\mathrm{N})$. These four functional proteins are responsible for host cell receptor binding, virion assembly, morphogenesis, and release of virus particles from the host cell[30].

The SARS-CoV-2 has a full size of 1,273 amino acids, longer than SARS-CoV (1,255 a.a), and it shares more than $90 \%$ amino acid identity SARS-CoV. However, phylogenetic-tree-based analysis suggests that SARS-CoV-2 is closer to SARS-like bat coronaviruses than to SARS-CoVs, specifically with the aspects of $\mathrm{S}$ gene sequence, mutation rate, and genetic diversity[30]. Thus, the SARS-CoV-2 virus has been officially classified into the subgenus Sarbecovirus of the Betacoronavirus genus[31].

\section{Symptoms}

\subsection{General Symptoms}

COVID 19 has different levels and types of influences to a different crowd. Most infected persons experience mild to moderate symptoms. Generally, symptoms appear 5-6 days after infection, but also possible 14 days after infection. The most common symptoms include fever, dry cough, and debilitation. In contrast, some unusual symptoms are ache, throat pain, diarrhea, conjunctivitis, headache, loss of taste or smell, rash, and discoloration of fingers or toes.

\subsection{Cytokine Storm and Respiratory Distress Syndrome}

Some COVID-19 patients encounter an acute respiratory distress syndrome with high mortality dependent on cytokine storm. Recently, increasing studies indicate that the cytokine storm may contribute to the mortality of COVID 19.

Cytokines are small glycoproteins produced by various types of cells throughout the body. The release 
of cytokines provides a wide range of functions, including regulating immune and inflammatory responses. Normally, cytokines are part of the body's immune response to infection, but their sudden release in large quantities can cause cytokine storm, a physiological reaction in the innate immune system. Cytokine storms can further stimulate the uncontrolled and excessive release of cytokines, which form a positive feedback loop, leading to multisystem organ failure and death[32].

During the COVID 19, SARS-CoV-2 activates the innate immune system and results in a release of a large number of cytokines, including IL-6, which can increase vascular permeability and cause a migration of fluid and blood cells into the alveoli and other consequent symptoms such as respiratory failure. One possible cause of cytokine storms in COVID 19 is the delayed-type I IFN response due to its accumulation of pathogenetic monocytes. Diabetes, hypertension, and cardiovascular disease are all risk factors of cytokine storms in COVID $19[33]$.

\section{Host}

\subsection{Bat}

Bat is an important natural host of alphacoronaviruses and beta coronaviruses. A bat coronavirus, named RaTG13, detected in Rhinolophus affinis from Yunnan Province, China, shows a similarity of $96.2 \%$ in fulllength genome sequence with SARS-CoV-2. And phylogenetic analysis confirms that SARS-CoV-2 closely clusters with RaTG13. Another novel bat virus denoted RmYN02, is 93.3\% identical to SARS-CoV-2 across the genome. In the long lab gene, it exhibits $97.2 \%$ similarity to SARS-CoV-2[29]. The high genetic similarity suggests that SARS-CoV-2 probably originates from the bat. However, current findings show that the divergence between SARS-CoV-2 and related bat coronaviruses likely represents more than 20 years of sequence evolution, suggesting that bat coronaviruses can only be the likely evolutionary precursor of SARS$\mathrm{CoV}-2$ rather than the direct progenitor.

\subsection{Pangolin}

Whether the pangolin participates in the transmission pf COVID-19 as an intermediate host has been debated since viral strains isolated from the infected Malayan pangolins shared $90.1 \%$ similarity with SARS-CoV-2 were found. Also, the E, M, N, and S genes encoding structural proteins between pangolin coronavirus and SARS-CoV-2 show 100\%, 98.6\%, 97.8\%, and 90.7\% amino acid similarity. Another independent sequence comparison detects a high similarity of $85.5 \%$ to $92.4 \%$ between pangolin coronavirus and SARS-CoV-2[34]. Besides, the phylogenetic tree confirms the close relation between pangolin coronavirus and SARS-CoV-2. However, pangolin didn't carry coronaviruses healthily. Infected pangolins showed clinical signs and histopathological changes, including interstitial pneumonia and inflammatory cell infiltration in diverse organs. What's more, several investigators found those published results above might originate from the same pangolin coronaviruses source[29, 35]. This suggests that pangolins are more likely to acquire the viruses after spillover from the natural host.

\section{Conclusion}

This review aims in presenting a comprehensive review on what we now know about COVID-19, including the pathogenesis, life cycle of the virus as well as the existing treatment methods. Considering the huge impact of COVID-19 pandemic, SARS-Cov-2 has been the hotspot of researchers all over the world and thanks to their devotion, we are rapidly unravelling the arcane mechanism of COVID-19 pathogenesis and potent vaccination strategies against COVID-19 have been developed at an unprecedentedly fast speed. Hopefully, we can get over this pandemic in the near future.

\section{References}

1. [COVID Live Update: 166,554,805 Cases and $3,459,730$ Deaths from the Coronavirus Worldometer [WWW Document]. [cited 2021 May 22]; Available from: https://www.worldometers.info/coronavirus/

2. Emerging Mutations \& Variants - CN [WWW Document]. [cited 2021 May 23]; Available from: www.thermofisher.cn/cn/zh/home/clinical/clinicalgenomics/pathogen-detection-solutions/covid-19sars-cov-2/mutations-variants.html.

3. Coronavirus (COVID-19) [WWW Document]. [cited 2021 May 23]; Available from: https://news.google.com/covid19/map?hl=enUS\&gl=US\&ceid=US:en.

4. Jin, Y., et al., Virology, Epidemiology, Pathogenesis, and Control of COVID-19. Viruses, 2020. 12(4): p. 372.

5. Tu, Y.F., et al., A Review of SARS-CoV-2 and the Ongoing Clinical Trials. International Journal of Molecular Sciences, 2020. 21(7): p. 2657.

6. Samad, N., et al., Convalescent Plasma Therapy for Management of COVID-19: Perspectives and Deployment in the Current Global Pandemic. Risk Management and Healthcare Policy, 2020. 13: p. 2707-2728.

7. Bracha, A. and S.Y. Tan, Emil von Behring (18541917): Medicine's first Nobel laureate. Singapore medical journal, 2011. 52(1): p. 1-2.

8. Winau, F. and R. Winau, Emil von Behring and serum therapy. Microbes \& Infection, 2002. 4(2): p. 185-188.

9. Behring and Kitasato, On the development of immunity to diphtheria and tetanus in animals. Dtsch Med Wochenschr, 1966. 90(49): p. 2183. 
10. Pucca, M.B., et al., History of Envenoming Therapy and Current Perspectives. other, 2019. 10.

11. Leider, J.P., P. Brunker, and P.M. Ness, Convalescent transfusion for pandemic influenza: preparing blood banks for a new plasma product? Transfusion, 2010. 50(6): p. 1384-1398.

12. Luke, T.C., et al., Meta-analysis: convalescent blood products for Spanish influenza pneumonia: a future H5N1 treatment? Ann Intern Med, 2006. 145(8): p. 599-609.

13. Fischer, J.C., et al., The role of passive immunization in the age of SARS-CoV-2: an update. European Journal of Medical Research, 2020. 25(1): p. 1-6.

14. Duan, K., et al., Effectiveness of convalescent plasma therapy in severe COVID-19 patients. Proceedings of the National Academy of Sciences, 2020. 117(17): p. 9490-9496.

15. Marano, G., et al., Convalescent plasma: new evidence for an old therapeutic tool? Blood Transfus, 2016. 14(2): p. 152-7.

16. Jiang, S., C. Hillyer, and L. Du, Neutralizing Antibodies against SARS-CoV-2 and Other Human Coronaviruses. Trends in Immunology, 2020. 41(5).

17. Takehiro, U., O. Kenji, and S. Masaru, Developments in Viral Vector-Based Vaccines. Vaccines, 2014. 2(3): p. 624-641.

18. Michael, et al., Sabin Vaccine Reversion in the Field: a Comprehensive Analysis of Sabin-Like Poliovirus Isolates in Nigeria. Journal of Virology, 2015.

19. Dediego, M.L., et al., A Severe Acute Respiratory Syndrome Coronavirus That Lacks the E Gene Is Attenuated In Vitro and In Vivo. Journal of Virology, 2006.

20. Macgregor, R.R., et al., First Human Trial of a DNA-Based Vaccine for Treatment of Human Immunodeficiency Virus Type 1 Infection: Safety and Host Response. Journal of Infectious Diseases, 1998. 178(1): p. 92-100.

21. Masters, P.S., The Molecular Biology of Coronaviruses, in Advances in Virus Research. 2006, Academic Press. p. 193-292.

22. Wu, S.-C., Progress and Concept for COVID-19 Vaccine Development. Biotechnology Journal, 2020. 15: p. 2000147.

23. Sks, A., et al., Prospect of SARS-CoV-2 spike protein: Potential role in vaccine and therapeutic development. Virus Research, 2020. 288.

24. Edison, O., et al., Vaxign-ML: Supervised Machine Learning Reverse Vaccinology Model for Improved Prediction of Bacterial Protective Antigens. Bioinformatics, 2020(10): p. 10.

25. Carlson, C.R., et al., Phosphoregulation of Phase Separation by the SARS-CoV-2 N Protein Suggests a Biophysical Basis for its Dual Functions. Molecular Cell, 2020. 80(6): p. 1092-1103.e4.
26. Keller, M.D., et al., SARS-CoV-2-specific T cells are rapidly expanded for therapeutic use and target conserved regions of the membrane protein. Blood, 2020. 136(25): p. 2905-2917.

27. Adedeji, B.J., et al., Therapeutic Drugs for SARSCoV-2 Treatment: Current State and Perspective. International Immunopharmacology, 2020.

28. Keyaerts, E., et al., In vitro inhibition of severe acute respiratory syndrome coronavirus by chloroquine. Biochemical and Biophysical Research Communications, 2004. 323(1): p. 264-268.

29. $\mathrm{Hu}, \mathrm{B}$., et al., Characteristics of SARS-CoV-2 and COVID-19. Nature Reviews Microbiology.

30. Mittal, A., et al., COVID-19 pandemic: Insights into structure, function, and hACE2 receptor recognition by SARS-CoV-2. PLoS Pathogens, 2020. 16(8): p. e1008762.

31. Izda, V., M.A. Jeffries, and A.H. Sawalha, COVID19: A review of therapeutic strategies and vaccine candidates. Clinical Immunology, 2021. 222: p. 108634.

32. Song, P., et al., Cytokine storm induced by SARSCoV-2. Clinica Chimica Acta, 2020. 509.

33. Mangalmurti, N. and C.A. Hunter, Cytokine Storms: Understanding COVID-19. Immunity, 2020. 53(1).

34. Rando, H.M., et al., Pathogenesis, Symptomatology, and Transmission of SARS-CoV-2 through analysis of Viral Genomics and Structure. 2021.

35. Tian, B.P., The potential intermediate hosts for SARS-CoV-2. Frontiers in Microbiology, 2020. 